University of New Hampshire

University of New Hampshire Scholars' Repository

Space Science Center

Institute for the Study of Earth, Oceans, and

Space (EOS)

2002

\title{
Readout and performance of thick CZT strip detectors with orthogonal coplanar anodes
}

John R. Macri

University of New Hampshire - Main Campus, John.Macri@unh.edu

B Donmez

University of New Hampshire - Main Campus

L A. Hamel

University of Montreal

Manuel Julien

University of Montreal

M McClish

University of New Hampshire - Main Campus

See next page for additional authors

Follow this and additional works at: https://scholars.unh.edu/ssc

Part of the Astrophysics and Astronomy Commons

\section{Recommended Citation}

Macri, J.R.; Donmez, B.; Hamel, L.-A.; Julien, M.; McClish, M.; McConnell, M.L.; Miller, R.S.; Ryan, J.M.; Widholm, M., "Readout and performance of thick CZT strip detectors with orthogonal coplanar anodes," Nuclear Science Symposium Conference Record, 2002 IEEE , vol.1, no., pp.468,472 vol.1, 10-16 Nov. 2002

This Conference Proceeding is brought to you for free and open access by the Institute for the Study of Earth, Oceans, and Space (EOS) at University of New Hampshire Scholars' Repository. It has been accepted for inclusion in Space Science Center by an authorized administrator of University of New Hampshire Scholars' Repository. For more information, please contact Scholarly.Communication@unh.edu. 


\section{Authors}

John R. Macri, B Donmez, L A. Hamel, Manuel Julien, M McClish, Mark L. McConnell, R S. Miller, James M. Ryan, and Mark Widholm 


\title{
Readout and Performance of Thick CZT Strip Detectors with Orthogonal Coplanar Anodes
}

\author{
John R. Macri, Burçin Dönmez, Louis-Andre Hamel, Manuel Julien, Mickel McClish, \\ Mark L. McConnell, Richard S. Miller, James M. Ryan, Mark Widholm
}

\begin{abstract}
We report progress in the study of CZT strip detectors featuring orthogonal coplanar anode contacts. The work includes laboratory and simulation studies aimed at optimizing and developing compact, efficient, high performance detector modules for 0.05 to $1 \mathrm{MeV}$ gamma radiation measurements. The novel coplanar anode strip configuration retains many of the performance advantages of pixel detectors yet requires far fewer electronic channels to perform both 3-d imaging and spectroscopy.

We report on studies aimed at determining an optimum configuration of the analog signal processing electronics to employ with these detectors. We report measurements of energy and spatial resolution in three dimensions for prototype 5 and $10 \mathrm{~mm}$ thick CZT detectors using a set of shaping and summing amplifiers.
\end{abstract}

\section{Orthogonal Coplanar AnOde Strip Detectors}

We have been studying a novel CZT detector configuration as an alternative to pixel and double-sided strip detectors [1][3]. Figure 1 illustrates the anode surface contact pattern and the readout of an $8 \times 8$ orthogonal coplanar anode strip detector. As with double-sided strip detectors, this detector requires

Manuscript received November 12, 2002. This work was supported by NASA's High Energy Astrophysics Supporting Research and Technology Program under Grant No. NAG5-5327.

J. R. Macri, member, IEEE, is with the University of New Hampshire Space Science Center, Durham, NH 03824 USA (telephone: (603) 862-2793, e-mail: john.macri@unh.edu).

M. L. McConnell, member, IEEE, is with the University of New Hampshire Space Science Center, Durham, NH 03824 USA (telephone: (603) 862-2047, e-mail: mark.mcconnell@unh.edu).

R. S. Miller is with the University of New Hampshire Space Science Center, Durham, NH 03824 USA (telephone: (603).862.3664, e-mail: richard.miller@unh.edu).

M. Widholm is with the University of New Hampshire Space Science Center, Durham, NH 03824 USA (telephone: (603) 862-4597, e-mail: mark.widholm@unh.edu).

J. M. Ryan, member, IEEE, is with the University of New Hampshire Space Science Center, Durham, NH 03824 USA (telephone: (603) 862.3510, e-mail: james.ryan@unh.edu).

B. Dönmez is a physics graduate student at the the University of New Hampshire, Durham, NH 03824 USA (telephone: (603) 862-4703, e-mail: bdonmez@cisunix.unh.edu).

M. McClish is a former UNH physics graduate student, currently at Radiation Measurement Devices, Watertown MA 02472 USA (telephone: (617) 926-1167, e-mail: MMcClish@rmdinc.com)

L. - A. Hamel A is with the physics department at Universite de Montreal, Montreal, Quebec, Canada, H3C-3J7. (telephone: (514) 343-6204, e-mail: hamel@lps.umontreal.ca).

M. Julien is a physics graduate student at Universite de Montreal, Montreal, Quebec, Canada, H3C-3J7. (telephone: (514) 343-6204, e-mail: julienman@yahoo.fr). simultaneous readout of both row and column signals. Each row takes the form of $\mathrm{N}$ discrete interconnected anode pixel electrodes while each column is a single strip electrode. (Fig. 1). The opposite surface, not shown, has a single uniform cathode electrode. The anode pixel contacts, interconnected in rows, are biased to collect the electron charge carriers. The orthogonal anode strips, surrounding the anode pixel electrodes, are biased between the cathode and anode pixel potentials. The strips register signals induced by the motion of electrons as they migrate to the pixels. Since electrons are much more mobile than holes in CZT, anode signals from photon interactions at all depths in the detector are detected, whereas with double-sided detectors hole trapping may attenuate or quench any cathode signal. The coplanar approach permits thicker, more efficient CZT imaging planes than are practical with double-sided strip detectors, extending the effective energy range to higher energies. In addition, more compact packaging is possible since all imaging contacts and signal processing electronics connections reside on one side of the detector.

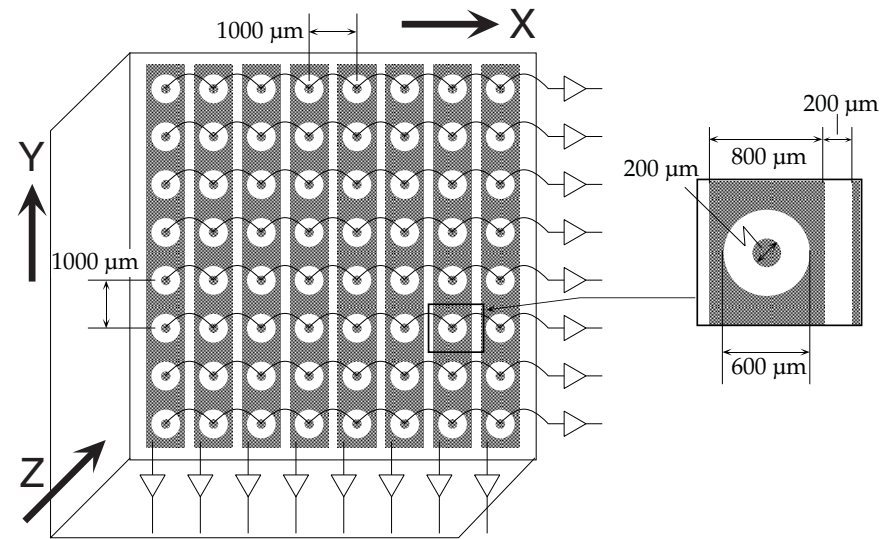

Fig. 1. Contact geometry and read out of the orthogonal coplanar anode design. Strip columns (X) are read out on the bottom. Pixel rows (Y) are read out on the right. The gold contact pattern dimensions shown here in grey correspond to those of our prototype assemblies.

Prototype detectors have been fabricated using material from both eV Products and Yinnel Tech, Inc. The CZT materials were processed and patterned by eV Products. Polymer flipchip bonding was used to form the electrical and mechanical connection of each patterned CZT substrate to its ceramic carrier. The result is a rugged detector module assembly that involves no wire bonds to the CZT anode surface [4]. $\quad$ Fig. 2 
shows photographs of two detector modules: Table 1 further identifies the prototype modules used in this study.
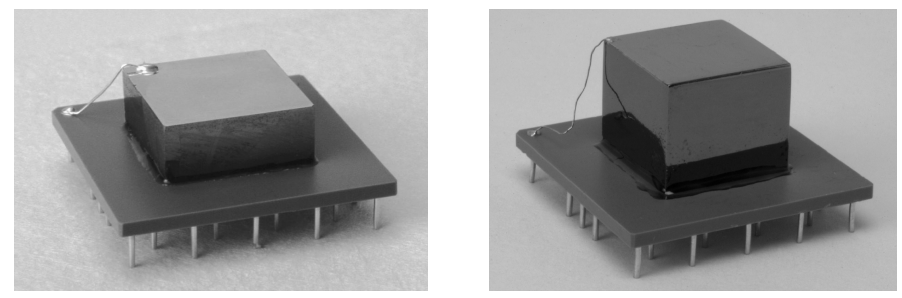

Fig. 2. Prototype detector modules: $5 \mathrm{~mm}$ thick (left), $10 \mathrm{~mm}$ thick (right).

TABLE I

Prototype Detector Modules

CZT thickness

Material

Assembly Date

UNH ID\#

Manufacturer

\begin{tabular}{cccc}
\hline $5 \mathrm{~mm}$ & eV Products & June, 1999 & UNH-EV-3 \\
$5 \mathrm{~mm}$ & eV Products & June, 1999 & UNH-EV-4 \\
$5 \mathrm{~mm}$ & Yinnel Tech. & Dec, 2001 & UNH-Y-5 \\
$10 \mathrm{~mm}$ & eV Products & Dec, 2001 & UNH-EV-11 \\
$10 \mathrm{~mm}$ & Yinnel Tech. & Dec, 2001 & UNH-Y-2 \\
\hline
\end{tabular}

\section{Measurement Setup}

Fig. 3 shows a block diagram of the signal processing.

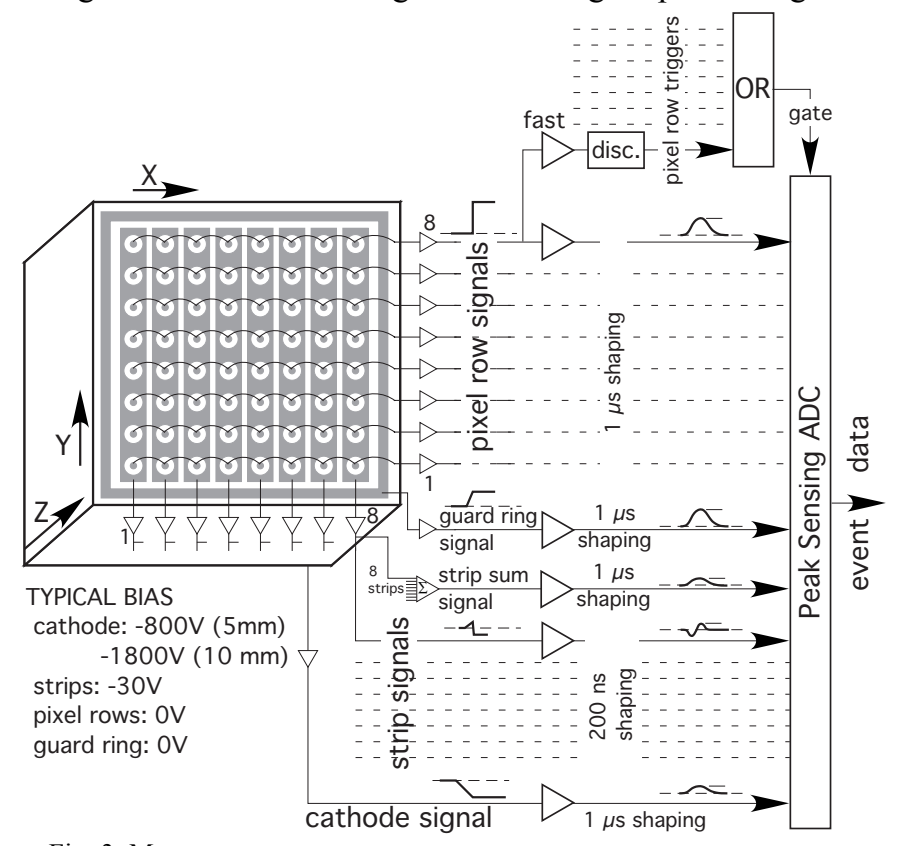

Fig. 3. Measurement setup.

The detectors were plugged into a custom test board for bias and readout of the charge signals with discrete preamplifiers (eV-5093). A strip sum signal is formed from the eight strip preamp outputs using a custom summing amplifier in the test box. The signal processing and data acquisition used NIM, CAMAC and VME electronics. Typical bias levels for the electrodes are shown for each detector thickness. Each charge collecting pixel row signal was split into a fast and a shaped channel. The fast pixel row channels were used to trigger event data acquisition. Any pixel row signal above its discriminator level will gate the peak sensing ADC. The peak level of 19 shaped signals ( 8 pixel rows, 8 orthogonal strips, cathode, strip sum and guard ring) was recorded for each triggered event. Note that different shaping times and polarities are selected for the various signal types.

\section{A. Measurement of Energy and the Y-Coordinate}

The small, charge collecting pixel row signals provide the best signal to noise ratio. As such they were used as the event trigger (fast channel) and to determine the energy and the $Y$ coordinate $(1 \mu$ s shaping) of the photon interaction location.

\section{B. Measurement of the X-Coordinate}

The strip signals were used to measure the $X$-coordinate of the photon interactions. The strip signal is generally bipolar and ranges in amplitude between $25 \%$ and $40 \%$ of the pixel signal. Ideally, the strips collect no charge, but, due to their size and proximity to the pixels, they do register the motion of charge in the detector and, specifically, of electrons as they are collected on the pixels. Fig.4 (left) shows simulated strip signals for several interaction depths in a $5 \mathrm{~mm}$ thick detector and illustrates several signal features. Fig. 4 (right) shows the relative signal to noise performance expected for several candidate shaping options. Note that while the strip signal shape changes significantly with the interaction depth, its negative "edge", as measured with $200 \mathrm{~ns}$ shaping, is present with reasonable signal-to-noise for all interaction depths. We used this shaping and converted the peak height of the second lobe of each shaped bipolar pulse (Fig. 3). These strip signal amplitudes were analyzed to determine the $X$-coordinate for each event, with the strip nearest the interaction registering the largest signal.
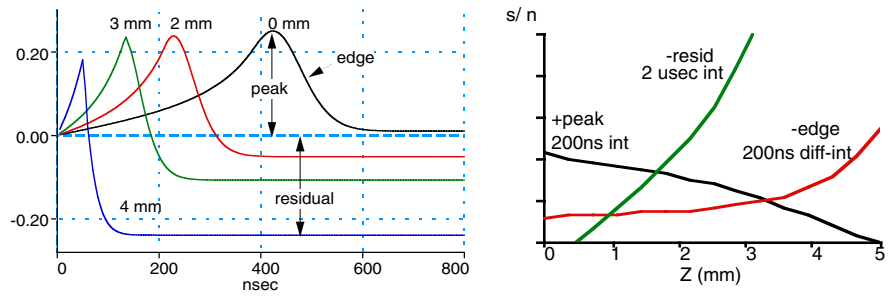

Fig. 4. Features of strip signals (simulated) at various depths (left). Simulated signal-to-noise ratio (relative units) vs. interaction depth for three signal processing options.

\section{Measurement of the Z-Coordinate, Interaction Depth}

Two independent measurements of the $Z$-coordinate were performed using methods incorporating the cathode and strip sum signals ( $1 \mu$ s shaping, Fig. 3$)$. This shaping for the strip sum signal is used to attain a measure of the depth-dependent residual strip signal as shown in Fig. 4.

\section{Performance}

\section{A. Spectroscopy}

A single pixel response to photons in the energy range from 14 to $662 \mathrm{keV}$ is shown in Figure 5. These data were collected in June 2002 using detector UNH-EV-4. This performanceis similar to that recorded in the initial testing of this detector in 1999 [1]. 

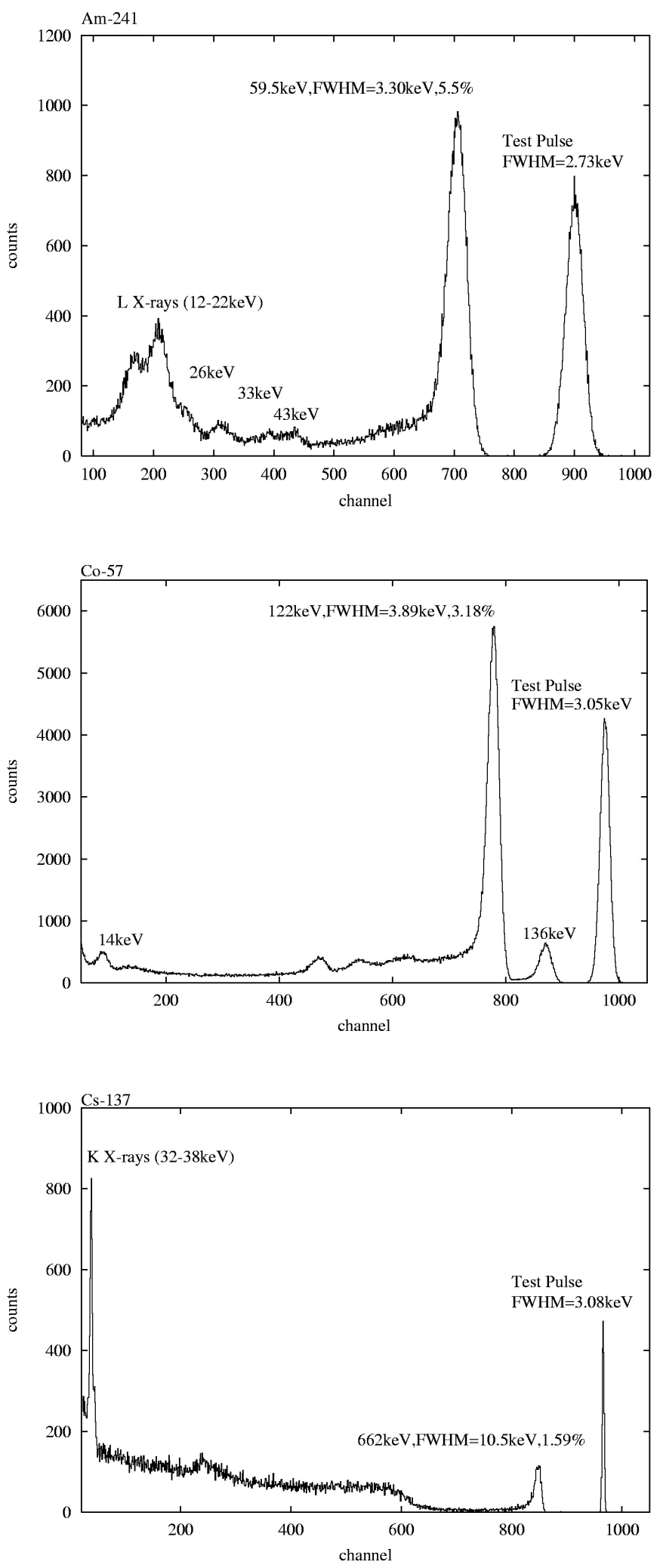

Fig. 5. Single "pixel" response across the energy range from 14 to 662 $\mathrm{keV}$ with $5 \mathrm{~mm}$ thick detector UNH-EV-4. Calibration sources: ${ }^{241} \mathrm{Am}$ (top), ${ }^{57} \mathrm{Co}$ (center), ${ }^{137} \mathrm{Cs}$ (bottom).
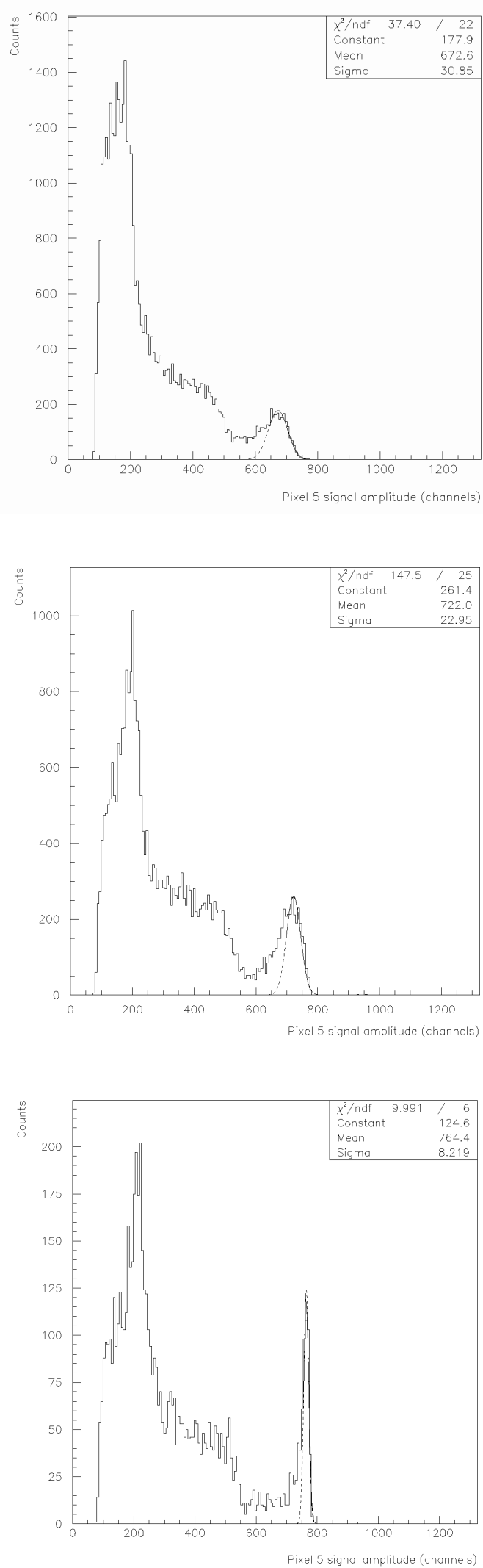

Fig. $6.10 \mathrm{~mm}$ thick detector UNH-EV-11 response to $662 \mathrm{keV}$ photons at different interaction depths: $Z=0-4.5 \mathrm{~mm}$ (top), $4.5-9 \mathrm{~mm}$ (middle), and 9-10 $\mathrm{mm}$ (bottom). 
The response to $662 \mathrm{keV}$ photons from a ${ }^{137} \mathrm{Cs}$ source of 10 $\mathrm{mm}$ thick detector UNH-EV-11 is shown in Fig. 6. The effect of electron trapping across the $10 \mathrm{~mm}$ thickness is evident in the photopeak pulse height as measured for different interaction depths $(Z)$. The best energy resolution $(2.5 \%$ FWHM) is measured for events occurring near the anode plane. In addition to the effects of electron trapping we have observed that a significant amount of charge is actually collected on the strips of this detector. This is not the case with our $5 \mathrm{~mm}$ thick detectors. Preliminary measurements indicate that $30 \%$ of events have more than $10 \%$ charge collection by the strip and that $15 \%$ of events have more than $50 \%$ charge collection by the strip. Further study is planned to better understand this effect. Since charge collected on the pixels is the measure of energy, this effect degrades spectroscopic performance. The charge collected on the strips has two other consequences: 1) it attenuates the pixel signal for some events dropping them below the trigger threshold resulting in a loss of efficiency and 2) it changes the nature of the induced signal on the strips thus degrading the resolution in the $X$ - and, eventually, $Z$ -

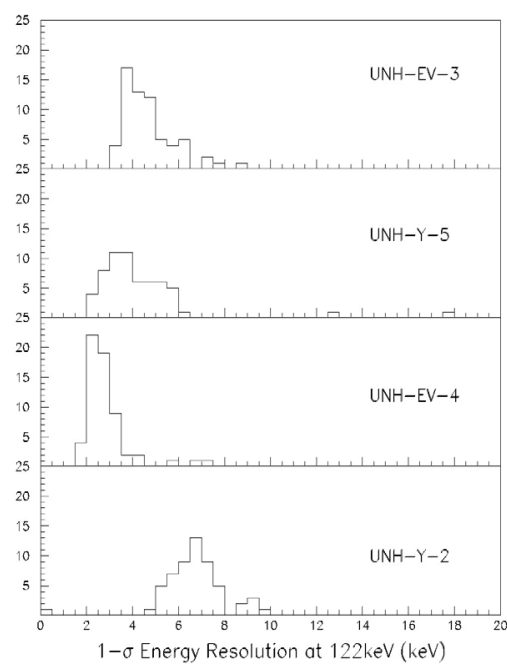

Fig. 7. Energy resolution distribution at $122 \mathrm{keV}$ for the 64 pixels of four prototype detectors (56 pixels are shown for UNH-Y2, bottom panel).

pixels) was too noisy to measure. No correction for electron trapping has been made here.

\section{B. Event location $(X, Y)$}

We used a tungsten collimator with a $0.2 \mathrm{~mm}$ wide bore hole and ${ }^{241} \mathrm{Am}$ and ${ }^{57} \mathrm{Co}$ point sources to measure the capability for determining the $X$ and $Y$ locations of photon interactions. Beam-spot scans were performed in $150 \mu \mathrm{m}$ steps $(X$ and $Y$ ) to calibrate a small region of $5 \mathrm{~mm}$ thick detector UNH-EV-3.

Figure 8 shows histograms of computed event locations $(X$, $Y$ ) in response to beam spot illuminations of this detector with $122 \mathrm{keV}$ and $60 \mathrm{keV}$ photons. The measured $1-\sigma$ spatial resolutions are $0.3 \mathrm{~mm}(X)$ and $0.2 \mathrm{~mm}(Y)$ at $122 \mathrm{keV}$ and $0.4 \mathrm{~mm}(X)$ and $0.2 \mathrm{~mm}(Y)$ at $60 \mathrm{keV}$. Sub-pitch (sub-mm) locations are computed by interpolation of the pulse height data of neighboring channels. Note that while the spatial resolution numbers in $Y$ are small, the computed locations in this dimension can be in error by as much as $0.5 \mathrm{~mm}$. This is a result of very little charge sharing between neighboring pixel rows. Signal to noise improvements of the strip signal circuitry are necessary to extend the threshold for measuring location in the $X$-dimension to lower energies.
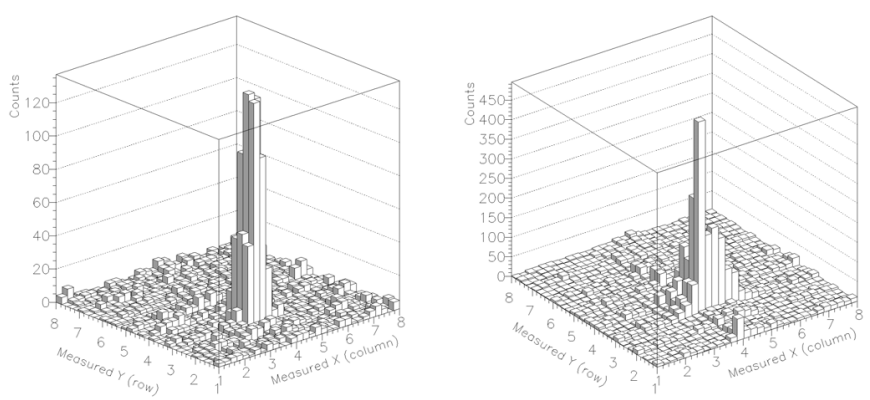

Figure 8. Beam spot images: $122 \mathrm{keV}$ (left), $60 \mathrm{keV}$ (right)

\section{Event location (Z), Depth of Interaction}

Depth calibration was obtained by illuminating detector UNH-EV-3 from the side, at a single depth, using $122 \mathrm{keV}$ photons from a ${ }^{57}$ Co source. A $0.4 \mathrm{~mm}$ wide slit in a tungsten block was used as the collimator. This slit collimator was used at seven different depths. Two methods were used to compute depth using the data: 1) cathode-to-maximum pixel ratio (c/a) and 2) strip sum-to-maximum pixel ratio (s/a). The $Z$-dependence was used to reconstruct the $Z$-coordinate by each method in analysis of each event in subsequent imaging demonstrations.

Fig. 9 shows the distribution of event locations for a slit beam incident normal to the full width of the $Y=0$ side of the detector at $Z=3.8 \mathrm{~mm}$. The interaction depth $(Z)$ was computed for each event using the cathode to maximum pixel ratio method. The measured $1-\sigma$ average spatial resolution at 122 $\mathrm{keV}$ across the full range of depth is $\sigma_{\mathrm{z}(\mathrm{c} / \mathrm{a})}=0.37 \mathrm{~mm}$ for the cathode to maximum anode pixel ratio method and $\sigma_{z(\mathrm{~s} / \mathrm{a})}=$ $0.86 \mathrm{~mm}$ for the strip sum to maximum anode pixel ratio method. The poorer result obtained using the strip sum method may be the result of summing the noise on the eight strip signals. While it meets our goal of $\sigma_{z}<1$ $\mathrm{mm}$, the resolution is

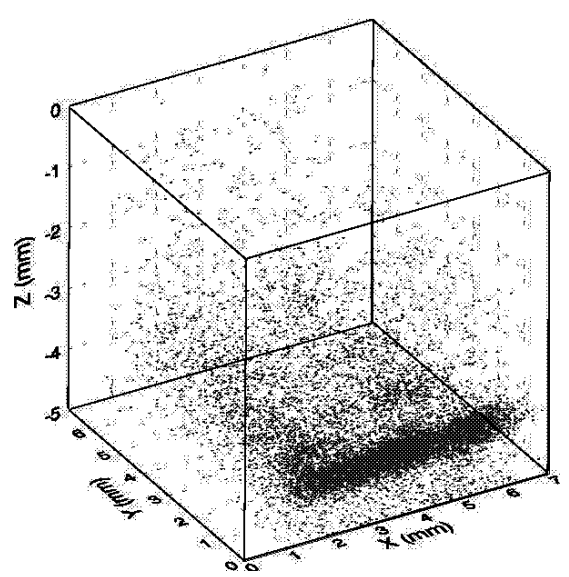

Fig. 9. Measurement of spatial resolution in $Z$. Illumination from the $\mathrm{Y}=0$ side through a 0.4 $\mathrm{mm}$ wide slit at $Z=3.8 \mathrm{~mm}$. 


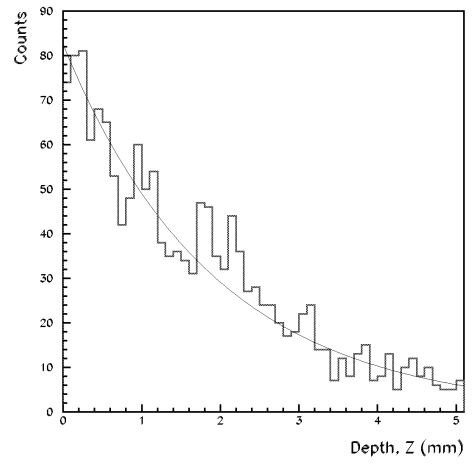

Fig. 10. Measurement of the attenuation length for $122 \mathrm{keV}$ photons in CZT.

Fig. 10 shows the measured distribution of interaction depths (Z) for $122 \mathrm{keV}$ photons from a ${ }^{57} \mathrm{Co}$ source illuminating the entire cathode surface at normal incidence. The attenuation length, $2.0 \pm 0.16 \mathrm{~mm}$, determined from a fit to the data, compares well with the theoretical value, 2.01 $\mathrm{mm}$.

\section{3-d Imaging}
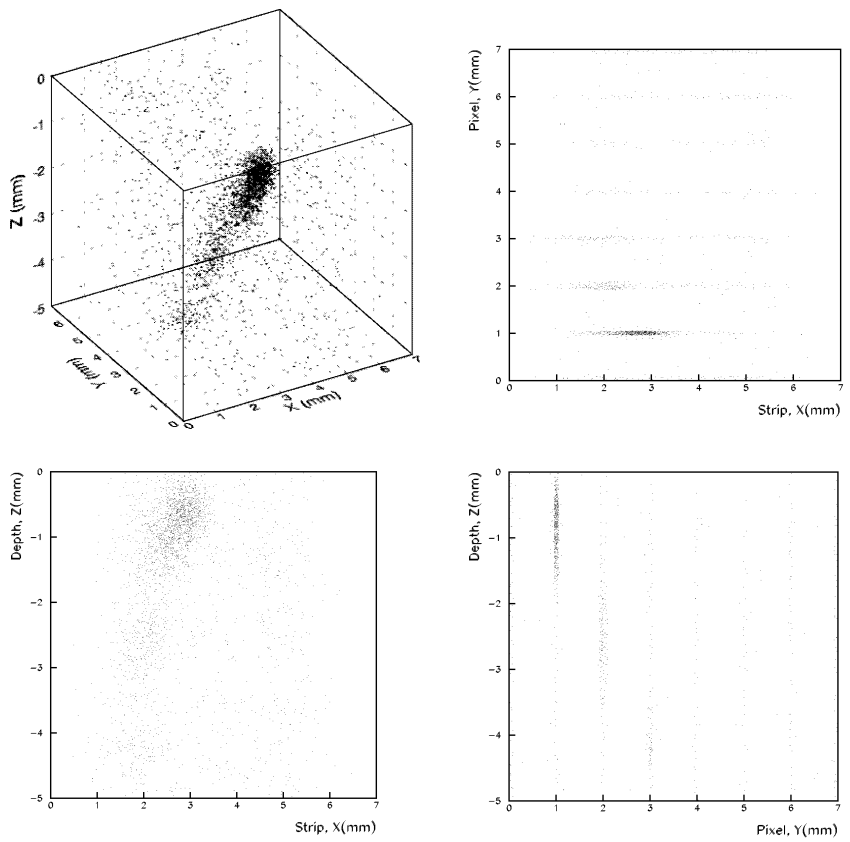

Fig. 11. Beam spot image, $\sim 25^{\circ}$ incidence. 3 -d event locations (top left), projections (clockwise) $x y, x z$ and yz. Note: cathode is at $Z=0$; sign of $Z$ was inverted to facilitate the graphic illustration.

Fig. 11 shows the $3-\mathrm{d}$ image and projections of a $0.2 \mathrm{~mm}$ beam spot of $122 \mathrm{keV}$ photons incident at $\sim 25^{\circ}$ from the $Z$ axis. The beam is directed so that it enters near strip 3, pixel 1 and crosses several pixel rows and several strip columns as it passes through the $5 \mathrm{~mm}$ thickness of the detector. The discontinuity of computed event locations in the $Y$-dimension indicates that there is very little charge sharing between pixel rows.

\section{CONCLUSIONS AND Future WORK}

We have employed relatively simple pulse shaping circuitry on signals of prototype 5 and $10 \mathrm{~mm}$ thick orthogonal coplanar anode CZT strip detectors and measured energy and spatial resolution performance in three dimensions. We have demonstrated good energy resolution in the range from 14 to $662 \mathrm{keV}$ and sub-mm imaging capabilities down to $60 \mathrm{keV}$ in the $X$ - and $Y$-coordinates and down to $122 \mathrm{keV}$ in the $Z$ coordinate using two methods.

Problems encountered include electron trapping and charge collection on the strip electrodes for the 10 $\mathrm{mm}$ thick detectors. Further study is required to better understand and address these issues. Simulations conducted at the University of Montreal suggest the anode pattern shown in Fig. 12 will help address the issues of discontinuous position

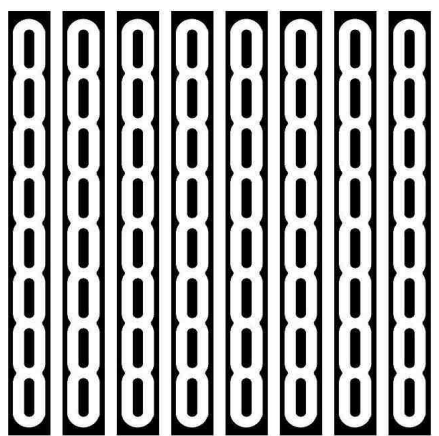

Figure 12. Alternative anode pattern under study with simulation tools. determination in the $Y$ -

dimension (charge sharing among neighboring pixels) and of electron trapping in $10 \mathrm{~mm}$ thick detectors. [3].

Future work will include the design, fabrication and test of prototype detector modules employing modified anode contact patterns as well as packaging and custom electronics development. Monte Carlo simulations will be developed to characterize the physics of high energy photon interactions in CZT.

\section{REFERENCES}

[1] M. L. McConnell, J. R. Macri, J. M. Ryan, K. Larson, L.-A. Hamel, G. Bernard, C. Pomerleau, O. Tousignant, J.-C. Leroux, V. Jordanov, Three-dimensional imaging and detection efficiency performance of orthogonal coplanar CZT strip detectors, Proc. SPIE, 4141, 157 (2000).

[2] J. M. Ryan, L. A. Hamel, M. Julien, J. R. Macri, M. McClish, M. L. McConnell, R. S. Miller and M. Widholm, "Development of CZT strip detector modules for $50 \mathrm{keV}$ to $1 \mathrm{MeV}$ imaging and Spectroscopy", Proc. SPIE, 4851 (2002)

[3] J. R. Macri, P. Dufour, L. A. Hamel, M. Julien, M. L. McConnell, M. McClish, J. M. Ryan, M. Widholm, "Study of 5 and $10 \mathrm{~mm}$ Thick CZT Strip Detectors," Conference Record, IEEE Nuclear Science Symposium, Nov. 2001.

[4] V.T. Jordanov, J.R. Macri, J.E. Clayton and K.A. Larson, MultiElectrode CZT Detector Packaging Using Polymer Flip Chip Bonding, NIM A 458, 511 (2001). 